INPLASY

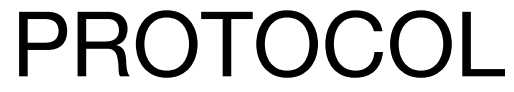

To cite: Yuan et al. A systematic review and metaanalysis of utility-based quality of life in chronic spontaneous urticaria. Inplasy protocol 202040038. doi:

10.37766/inplasy2020.4.0038

Received: 07 April 2020

Published: 07 April 2020

Corresponding author: Yan Yuan

yy12267708@163.com

Author Affiliation:

Department of Dermatology,

Xiangya Hospital, Centr

Support: National Natural

Science Found

Review Stage at time of this submission: Data analysis.

Conflicts of interest: No.

\section{A systematic review and meta- analysis of utility-based quality of life in chronic spontaneous urticaria}

\author{
Yuan, Y1; Xiao, Y2; Chen, $X^{3}$; Li, J4; Shen, M ${ }^{5}$
}

Review question / Objective:

The meta-analysis and systematic literature review of published data is intended to systematically determine pooled estimates of utility-based quality of life in patients with CSU. Condition being studied: Chronic urticaria (CU) is one of the most common skin disorders characterized by the rapid appearance of wheals, angioedema, or both, for more than 6 weeks. The symptoms of $\mathrm{CU}$ are caused by the activation of skin mast cells and subsequent histamine release and other inflammatory mediators. The annual prevalence of $\mathrm{CU}$ is estimated to be $0.1-1 \%$ in the general population. Quality of life estimates (utilities) is essential for economic evaluation because the quality of life is a critical component of economic benefits. Quality-adjusted life years are a measure of a person's length of life, which is an assessment of their healthrelated quality of life during that time. The purpose of this study is to systematically evaluate the methods and results of the health utility value of CSU-related disease states, and to provide data reference for subsequent utility evaluation.

INPLASY registration number: This protocol was registered with the International Platform of Registered Systematic Review and Meta-Analysis Protocols (INPLASY) on 07 April 2020 and was last updated on 07 April 2020 (registration number INPLASY202040038).

\section{INTRODUCTION}

Review question / Objective: The metaanalysis and systematic literature review of published data is intended to systematically determine pooled estimates of utility-based quality of life in patients with CSU.
Condition being studied: Chronic urticaria (CU) is one of the most common skin disorders characterized by the rapid appearance of wheals, angioedema, or both, for more than 6 weeks. The symptoms of $\mathrm{CU}$ are caused by the activation of skin mast cells and subsequent histamine release and other inflammatory mediators. The annual 
prevalence of $\mathrm{CU}$ is estimated to be $0.1-1 \%$ in the general population. Quality of life estimates (utilities) is essential for economic evaluation because the quality of life is a critical component of economic benefits. Quality-adjusted life years are a measure of a person's length of life, which is an assessment of their health-related quality of life during that time. The purpose of this study is to systematically evaluate the methods and results of the health utility value of CSU-related disease states, and to provide data reference for subsequent utility evaluation.

\section{METHODS}

Participant or population: Chronic urticaria (CU) is one of the most common skin disorders characterized by the rapid appearance of wheals, angioedema, or both, for more than 6 weeks. The symptoms of $\mathrm{CU}$ are caused by the activation of skin mast cells and subsequent histamine release and other inflammatory mediators. The annual prevalence of $\mathrm{CU}$ is estimated to be $0.1-1 \%$ in the general population. Quality of life estimates (utilities) is essential for economic evaluation because the quality of life is a critical component of economic benefits. Quality-adjusted life years are a measure of a person's length of life, which is an assessment of their health-related quality of life during that time. The purpose of this study is to systematically evaluate the methods and results of the health utility value of CSU-related disease states, and to provide data reference for subsequent utility evaluation.

Intervention: Exposure is defined as a clinical diagnosis of chronic spontaneous urticaria. Chronic spontaneous urticaria (CSU) is one of the most common skin disorders characterized by the rapid appearance of wheals, angioedema, or both, for more than 6 weeks.

Comparator: Where relevant, give details of the alternatives against which the main subject/topic of the review will be compared (e.g. another intervention or a non-exposed control group). The preferred format includes details of both inclusion and exclusion criteria.

Study designs to be included: Where relevant, give details of the alternatives against which the main subject/topic of the review will be compared (e.g. another intervention or a $n$

Eligibility criteria: Where relevant, give details of the alternatives against which the main subject/topic of the review will be compared (e.g. another intervention or a non-exposed control group). The preferred format includes details of both inclusion and exclusion criteria.

Information sources: We searched PubMed, Embase, Web of Science, the Cochrane Database of Systematic Reviews.Including full-text articles in both English and Chinese. We then conducted a meta-analysis that included all of these studies published from database inception to 31 April 2019. The search strategy adjusts to a controlled vocabulary for each database. We also reviewed references in groundbreaking essays, review articles, and medical textbooks. Gray literature and conference abstracts have not been searched.

Main outcome(s): The main result is the utility value quality of life, which is measured by the utility method.

\section{Additional outcome(s): None.}

Quality assessment / Risk of bias analysis: The quality assessment of included casecontrol studies and cross-sectional studies was assessed using the Newcastle-Ottawa Scale (NOS). The Newcastle-Ottawa Scale includes a series of questions that are used to assess the choice of study participants, the comparability of the population, and the determination of exposure or outcomes with a maximum score of 9 . Studies with scores greater than or equal to 5 were classified as high quality studies. Publication bias was assessed by the funnel plot. 
Strategy of data synthesis: The command "metan" was used to conduct metaanalysis using the STATA version 14 . Heterogeneity among the studies was evaluated using the $I^{2}$ statistic. Fixed effect model was used if $\mathrm{I}^{2}<50 \%$. Pooled utility estimates and $95 \%$ confidence intervals (CIs) were calculated and displayed as forest plots. Significance of subgroups was determined by Wald test. Meta regression was performed to examine the effect of gender on utility estimates. Publication bias may arise in this field of investigation, so using the Begg and Egger's tests used for publication bias.

Subgroup analysis: In subgroup analysis, utility elicitation approaches were evaluated. In sensitivity analysis, one study was eliminated at a time and the pooled estimate was calculated.

Sensibility analysis: The quality assessment of included case-control studies and crosssectional studies was assessed using the Newcastle-Ottawa Scale (NOS). The Newcastle-Ottawa Scale includes a series of questions that are used to assess the choice of study participants, the comparability of the population, and the determination of exposure or outcomes with a maximum score of 9. Studies with scores greater than or equal to 5 were classified as high quality studies. Publication bias was assessed by the funnel plot.

Country(ies) involved: China.

Keywords: Chronic spontaneous urticaria; utility-estimate; disease burden. 